\title{
Impactos do agrupamento do bambu Actinocladum verticillatum (Nees) McClure ex Soderstr. (POACEAE) sobre a vegetação lenhosa de duas fitofisionomias de Cerrado na transição Cerrado-Floresta Amazônica
}

\begin{abstract}
Divino Vicente SILVÉRIO ${ }^{1}$, Henrique Augusto MEWS², Eddie LENZA³ ${ }^{3}$ Beatriz Schwantes MARIMON ${ }^{4}$
RESUMO

Este estudo analisou os efeitos do agrupamento de uma espécie de bambu nativa do Cerrado (Actinocladum verticillatum) sobre a composiçáo florística, diversidade e estrutura da vegetação lenhosa de um cerradão e de um cerrado típico adjacentes no Parque Municipal do Bacaba, Nova Xavantina-MT. Foram instaladas 60 parcelas de 10x10 m, sendo 30 no cerradão e 30 no cerrado típico. Em cada fitofisionomia, foram instaladas 15 parcelas em um sítio sem bambu (SB) e 15 em um sítio com cobertura estimada de folhagem de bambu superior a 90\% (CB). Foram amostrados todos os indivíduos arbustivo-arbóreos com diâmetro a 30 centímetros do solo (DAS) $\geq 3 \mathrm{~cm}$. Foi avaliada a riqueza, a diversidade de espécies, a similaridade florística, a distribuição de diâmetros e alturas e o índice de valor de importância das espécies (VI). De forma geral, os sítios CB das duas fitofisionomias apresentaram menores valores quanto ao número de indivíduos, espécies, gêneros, famílias, densidades e áreas basais em relação aos sítios $\mathrm{SB}$, com redução mais acentuada nestes parâmetros no cerrado típico em relação ao cerradão. Os resultados sugerem que a ocupação do espaço e a redução da incidência luminosa causada pelas touceiras do bambu dificultam a germinação das sementes e o estabelecimento das plântulas de espécies arbustivo-arbóreas, selecionando as espécies mais tolerantes ao sombreamento modificando a composição florística e a estrutura da vegetação.
\end{abstract}

PALAVRAS-CHAVE: Bambu, competição, conservação, estruturação da comunidade, espécies lenhosas.

\section{Impact of Actinocladum verticillatum (Nees) McClure ex Soderstr. (POACEAE) clumps on the woody vegetation in two Cerrado physiognomies in transition Cerrado - Floresta Amazônica, east of Mato Grosso, Brazil}

\begin{abstract}
This study evaluated the effects of a Cerrado native bamboo species (Actinocladum verticillatum) clumps on the floristic composition, diversity and structure of the woody vegetation in two adjacent areas of "cerradão" and typical "cerrado" in the Bacaba Municipal Park, Nova Xavantina, state of Mato Grosso, Brazil. Sixty plots of 10 x 10 m were established, 30 in each forest physiognomy. In each physiognomy, 15 plots were placed in a bamboo-free site (SB) and 15 in a site with an estimated bamboo leaf cover of over 90\% (CB). All individuals (shrubs and trees) with a diameter at soil height (DSH $=30$ $\mathrm{cm}$ aboveground) $\geq 3 \mathrm{~cm}$ were sampled. Species richness and diversity, floristic similarity, diameter and height distribution, and the species importance value index (IVI) were evaluated. In general, CB sites of both physiognomies had lower values of number of individuals, species, genera, families, density and basal area compared to SB sites, with marked decrease in these parameters in the cerrado in relation to cerradáo. Results suggest that the space occupation and reduction of light incidence caused by bamboo clumping affect seed germination and seedling establishment of Cerrado shrubs and trees, selecting more shade-tolerant species and changing the floristic composition and vegetation structure.
\end{abstract}

KEYWORDS: bamboo, competition, conservation, community structure, woody species.

\footnotetext{
1 Universidade do Estado de Mato Grosso.E-mail: vicentth@yahoo.com.br

2 Universidade do Estado de Mato Grosso.E-mail: henriquemews@gmail.com

3 Universidade do Estado de Mato Grosso.E-mail: eddielenza@yahoo.com.br

${ }^{4}$ Universidade do Estado de Mato Grosso.E-mail: biamarimon@hotmail.com
} 


\section{INTRODUÇÃO}

O leste do estado de Mato Grosso engloba uma zona de tensão ecológica entre os biomas Cerrado e Floresta Amazônica e apresenta um mosaico vegetacional com domínio de fitofisionomias savânicas do bioma Cerrado (Ratter et al. 1973; Ivanauskas et al. 2004; Marimon et al. 2006). Inserida no "arco do desmatamento", esta região é conhecida pelas elevadas taxas de perda de floresta, o que tem levado à degradação dos ecossistemas e à perda de biodiversidade (Nepstad et al. 1999; Fearnside 2005).

As florestas mais periféricas da Amazônia apresentam menor biomassa principalmente devido ao menor porte da floresta e menor densidade da madeira, sendo que estas diferenças são ainda mais acentuadas para as florestas que apresentam alta densidade de bambus (Nogueira et al. 2008).

Além de diminuir a produtividade, a alta densidade de bambus também está relacionada à perda de biodiversidade em ecossistemas tropicais, como na floresta amazônica no Acre e na floresta semidecidual em Minas Gerais. Silveira (2001) observou que a presença de bambus da espécie Guadua weberbaueri pode influenciar a germinação de sementes e o estabelecimento de plântulas das espécies arbóreas no estado do Acre. Oliveira-Filho et al. (1994) verificaram que nas áreas com adensamento de bambu da espécie Merostachys neesii, as espécies arbóreas de uma floresta semidecídua no sudeste do Brasil apresentaram baixa densidade e área basal e concluíram que os bambus dificultam o recrutamento dessas espécies por interferir nos processos de germinação e estabelecimento das plântulas. Sanquetta et al. (2006) analisaram o estabelecimento de plântulas de espécies arbóreas de uma floresta ombrófila mista montana no Paraná e concluíram que o corte dos bambus resultou no alto recrutamento de espécies arbóreas.

A maioria das espécies de bambu são semelparas (um único evento de reprodução sexuada), reproduzindo-se em intervalos que podem variar de poucos anos a mais de um século. Nesse intervalo de tempo elas se multiplicam vegetativamente através de rizomas (Janzen 1976), apresentando rápido crescimento dos colmos e densa folhagem, tornando-se altamente competidoras por espaço e por luz (Silveira 2001; OliveiraFilho et al. 2004).

No Parque Municipal do Bacaba, em Nova XavantinaMT, a espécie arbustiva de bambu Actinocladum verticillatum ocorre em densos agrupamentos em diferentes fisionomias de vegetação, como cerrado típico, cerradão e mata de galeria. $A$. verticillatum é uma espécie nativa de bambu (Mendonça et al. 2008), mas a ocorrência de distúrbios como o fogo pode afetar os processos demográficos das populaçóes desta espécie, podendo levar a um aumento na sua área de abrangência e densidade local (Soderstrom 1981).
Pouco se sabe sobre os efeitos dos agrupamentos de bambus sobre a composição florística e estrutura da comunidade lenhosa. Ademais, considerando que os bambus são altamente competidores por espaço e por luz (Silveira 2001; OliveiraFilho et al. 2004), espera-se que seus efeitos sejam maiores em ambientes savânicos e mais iluminados, como o cerrado típico, que apresenta cobertura arbórea de 20 a 50\% e altura média de 3 a $6 \mathrm{~m}$, do que em ambientes florestais mais sombreados, como o cerradão, que apresenta dossel quase contínuo, com cobertura arbórea de 50 a $90 \%$ e altura média de 8 a 15 m (Ribeiro e Walter 2008).

Nesse sentido, destaca-se a importância da execução deste estudo para uma avaliação dos impactos do agrupamento de A. verticillatum sobre a composiçáo florística, a diversidade de espécies e a estrutura da vegetação lenhosa de duas áreas adjacentes das fitofisionomias cerradão e cerrado típico.

\section{MATERIAL E MÉTODOS}

\section{ÁREA DE ESTUDO}

O estudo foi desenvolvido no Parque Municipal do Bacaba, uma unidade de conservaçáo municipal com uma área de 492 hectares. O Parque, situado no município de Nova Xavantina (14\% $42^{\prime} 28,8^{\prime \prime}$ S e $52^{\circ} 21^{\prime} 03,9^{\prime \prime}$ W), na regiáo leste de Mato Grosso, apresenta várias fisionomias de vegetação (Abad e Marimon 2008). Os solos da área de estudo são predominantemente distróficos, álicos, ácidos, profundos, bem drenados, de textura média e com reduzidos níveis de cálcio e magnésio nas camadas superficiais (Marimon Júnior e Haridasan 2005). O clima da região é do tipo $A w$ de Köppen (Camargo, 1963), com duas estaçóes bem definidas, sendo o período seco de maio até setembro e o chuvoso de outubro a março. A precipitação média anual, segundo dados coletados junto à Estação Meteorológica localizada no Parque Municipal do Bacaba em Nova Xavantina, é de $1.536 \mathrm{~mm}$, com médias de temperaturas em torno de $25,5^{\circ} \mathrm{C}$ (Marimon e Felfili, 2006).

\section{DESCRIÇÃO DA ESPÉCIE}

Nas áreas de cerrado e cerradão, é comum a ocorrência de adensamentos naturais de Actinocladum verticillatum. Esta espécie possui rizomas cobertos por duras escamas protetoras contra o dessecamento e o fogo, que promovem a reproduçáo vegetativa e garantem a produção de novos caules aéreos após a ocorrência de queimadas. Estas características permitem o aumento nos adensamentos das populaçóes de $A$. verticillatum após a passagem do fogo (Soderstrom 1981).

De acordo com Filgueiras (1988), A. verticillatum cresce em touceiras vigorosas que podem alcançar até quatro metros de altura, possuindo rizomas do tipo paquiforme e folhas de três tipos, sendo as do ápice e do colmo grandes e largas e em número reduzido e as folhas situadas em complementos 
de ramos lateralmente ao nó são verticiladas, estreitas e numerosas. $\mathrm{O}$ referido autor observa ainda que a espécie é considerada uma excelente forrageira nativa para a pecuária, visto que suas folhas apresentam cerca de $12 \%$ de proteína, representando um recurso importante, especialmente na estaçáo seca, quando suas folhas permanecem verdes.

\section{DELINEAMENTO EXPERIMENTAL}

Para a execuçáo do presente estudo foram estabelecidas 60 parcelas de 10x10 m, sendo 30 numa área de cerradáo e 30 em um cerrado típico (de acordo com Ribeiro e Walter 2008). Em cada fitofisionomia foram estabelecidas 15 parcelas contíguas em um sítio controle, com ausência completa de bambu (SB), e 15 parcelas contíguas em um sítio adjacente com intenso agrupamento de $A$. verticillatum (CB), onde a cobertura da folhagem de bambu, estimada visualmente, era superior a $90 \%$. As áreas de cerradáo e cerrado típico distam cerca de 600 metros entre si e as parcelas dos sítios SB e CB dentro da mesma fitofisionomia distam 20 metros entre si.

Foram amostrados, dentro das parcelas, todos os indivíduos lenhosos vivos e mortos que apresentaram diâmetro igual ou superior a $3 \mathrm{~cm}$, medido a $30 \mathrm{~cm}$ do solo $\left(\mathrm{DAS}_{30} \geq\right.$ $3 \mathrm{~cm}$ ). Os indivíduos que apresentaram ramificaçóes tiveram todos os ramos medidos e foi calculado o diâmetro quadrático, conforme proposto por Scolforo (1993). Para a classificação das famílias botânicas, foi utilizado o sistema APG II (2003) e a revisão e atualizaçáo dos nomes dos táxons foi baseada em Mobot (2009).

\section{ANÁLISES DOS DADOS}

A diversidade de espécies para cada fitofisionomia foi calculada a partir do índice de Shannon-Wiener (H') e comparada entre sítios (CB e SB) da mesma fitofisionomia com o uso do teste $t$ de Hutcheson (Zar 1999). Para calcular a uniformidade (equabilidade) foi utilizado o índice de Pielou (J') e a similaridade florística entre sítios da mesma fitofisionomia e de fitofisiomias diferentes foi calculada através do índice de Jaccard $\left(\mathrm{S}_{\mathrm{J}}\right)$. Para os cálculos, foi utilizado o programa Mata Nativa 2.09 (Cientec 2002), sendo as equaçóes baseadas em Brower e Zar (1977) e Ludwing e Reynolds (1988). A densidade de indivíduos e a frequência de espécies exclusivas foi comparada entre sítios da mesma fitofisionomia por meio do teste de qui-quadrado $\left(\chi^{2}\right)$ (Zar 1999).

Para analisar a riqueza de espécies, nas duas fitofisionomias, foi aplicado o método de curvas de rarefação de espécies (Krebs 2000), com o programa EstimateS 8.0 (Colwell 2008) e construídas a partir de 1000 aleatorizaçóes. Foi utilizado o estimador Jackknife 1 para estimar o número de espécies que poderiam ser amostradas caso a amostragem fosse intensificada (Magurran 1988). A riqueza de espécies entre sítios na mesma fitofisionomia foi comparada pelo método de rarefação com o programa ECOSIM 7.0 (Gotelli e Entsminger 2001). Este programa permite fixar um número de indivíduos menor do que o observado em uma amostra e estima a riqueza para este número de indivíduos retirado aleatoriamente da amostra. Para gerar as estimativas, foram realizadas 1000 aleatorizaçóes e utilizado o intervalo de confiança de $95 \%$. A partir da média de espécie e variância fornecida pelo programa foi calculado um valor de $p$ com base no teste $Z$ de distribuição normal (Zar 1999).

Foram determinadas as distribuiçôes em classes de altura e diâmetro das duas fitofisionomias nos sítios com e sem bambu. Os intervalos de classes (IC), tanto para as alturas quanto para os diâmetros, foram calculados a partir da fórmula proposta por Spiegel (1976) e as comparaçôes das distribuições de altura e de diâmetro foram realizadas através do teste de KolmogorovSmirnov (Siegel e Castellan-Júnior 1988).

Para a descrição da estrutura da vegetação foi calculado o índice de valor de importância (VI), conforme proposto por Curtis e McIntosh (1950, 1951) e Müeller-Dombois e Ellemberg (1974). Para os cálculos foi utilizado o programa Mata Nativa 2.09 (Cientec 2002). Adicionalmente, foi aplicado o método de classificação TWINSPAN (Two-Way Indicator Species Analysis) para identificar agrupamentos de espécies em funçấo das parcelas (unidades amostrais) com características florísticas comuns (Hill 1979; Kent e Cocker 1992). Os resultados do TWINSPAN mostram as divisōes e as espécies indicadoras e preferenciais das unidades amostrais (Felfili et al. 2007).

\section{RESULTADOS}

Em geral, os sítios CB de cerrado e cerradáo apresentaram menores valores quanto ao número de indivíduos, espécies, gêneros, famílias e área basal, quando comparados aos sítios $\mathrm{SB}$, com tendências para reduçóes mais acentuadas no cerrado típico em relação ao cerradão (Tabela 1). Os sítios $\mathrm{SB}$ apresentaram maiores densidades do que os sítios $\mathrm{CB}$ (cerradâo $-\chi_{1,0,05}^{2}=27,90, p<0,001$; cerrado típico $-\chi_{1,0,05}^{2}$ $=398,06, p<0,001)$. Houve ainda uma tendência de menor área basal nos sítios CB para as duas fitofisionomias (15\% menor para o cerradão e $28 \%$ menor para o cerrado típico).

As curvas de rarefação mostraram, tanto para o cerradão quanto para o cerrado típico, uma tendência à estabilidade (Figura 1), indicando que a área amostrada foi suficiente para contemplar a maioria das espécies do ambiente. A riqueza registrada de espécies foi maior nos sítios SB; no entanto, as diferenças foram mais evidentes no cerrado típico do que no cerradáo (vide também Tabela 1). A riqueza estimada de espécies foi sempre maior no sítio sem bambu dentro da mesma fitofisionomia (Tabela 1). Considerando o mesmo número de indivíduos para efeito de ajuste do esforço amostral, no sítio sem bambu do cerradão seriam amostradas 69 espécies, 
não diferindo do sítio com bambu (67) $(z=-0,839 ; \mathrm{p}=0,200)$. Já no sítio sem bambu do cerrado seriam amostradas 70 espécies, sendo maior que aquela riqueza observada no sítio com bambu desta fitofisionomia (64) $(z=-1,749 ; \mathrm{p}=0,040)$.

No cerrado típico a frequência de espécies exclusivas no sítio SB foi maior do que no sítio CB $\left(\chi_{1,0.05}^{2}=20,16, p<\right.$ $0,001)$ e não houve diferença significativa na frequência de espécies exclusivas entre os sítios $\mathrm{SB}$ e CB do cerradão $\left(\chi^{2}{ }_{10,05}\right.$ $=1,18 ; \mathrm{p}=0,27)$. A diversidade de espécies do cerrado típico foi maior no sítio SB do que no sítio $\mathrm{CB}(t=5,07 ; p<0,001)$, enquanto no cerradão os valores de $\mathrm{H}^{\prime}$ não diferiram entre os dois sítios $(t=0,43 ; p>0,05)$. Os sítios $\mathrm{SB}$ e CB dentro da mesma fitofisionomia apresentaram alta similaridade florística (45\% no cerradão e 59\% no cerrado típico) (Tabela 1). A similaridade entre os sítios $\mathrm{CB}$ das duas fitofisionomias também foi elevada (61\%), enquanto que entre sítios SB das duas fitofisionomias foi menor (42\%). Todos os sítios das duas fitofisionomias apresentaram equabilidade elevada e semelhante, apontando uma distribuição equânime dos indivíduos entre as espécies (Tabela 1).

A distribuição de alturas dos indivíduos lenhosos dos dois sítios, para as duas fitofisionomias, apresentou maior número de indivíduos nas classes intermediárias, com um padrão tendendo a distribuição normal (Figura 2). No entanto, para o cerradão a distribuição dos indivíduos entre as classes de altura foi diferente entre os sítios SB e CB $\left(\chi^{2}=13,78\right.$, $p$ $=0,001)$, com uma tendência para maiores frequências nas classes inferiores no sítio $\mathrm{CB}$. Os sítios $\mathrm{SB}$ e CB do cerrado típico também diferiram quanto à distribuição dos indivíduos nas classes de altura $\left(\chi^{2}=11,99, p=0,0025\right)$; no entanto, nesta fitofisionomia foi observada uma tendência para frequências maiores nas últimas classes de altura no sítio CB (Figura 2).

No cerradão, não houve diferença significativa entre os sítios SB e CB nas freqüências de distribuição de indivíduos nos intervalos de classes de diâmetro $\left(\chi^{2}=1,17, p=0,55\right)$. Entretanto, no cerrado típico houve diferença significativa para a distribuiçáo de diâmetros entre os dois sítios $\left(\chi^{2}=32,22\right.$, $p=0,0001)$, devido principalmente às maiores frequências relativas de indivíduos nas classes superiores no sítio $\mathrm{CB}$ e à maior freqüência de indivíduos na menor classe no sítio $\mathrm{SB}$ (Figura 3).

No total, foram amostradas 125 espécies nos quatro sítios das duas fitofisionomias. No sítio SB do cerradão, as espécies Sclerolobium paniculatum, Myrcia sellowiana, Hirtella glandulosa, Eriotheca gracilipes e Matayba guianensis se destacaram em termos de VI e representaram $49 \%$ do VI total. Por outro lado, no sítio $\mathrm{CB}$ as espécies de destaque foram Sclerolobium paniculatum, Roupala montana, Emmotum nitens, Banisteriopsis pubipetala e Xylopia aromatica, representando $38 \%$ do VI total (veja apêndice disponível on-line no site http://www.scielo.br/aa). No sítio SB do cerrado típico, Banisteriopsis pubipetala, Davilla elliptica, Roupala montana, Myrcia sellowiana e Matayba guianensis representaram $21 \%$ do VI total. As espécies principais do sítio CB do cerrado típico foram Emmotum nitens, Curatella americana, Terminalia argentea, Eriotheca gracilipes e Syagrus flexuosa, que representaram 29\% do VI total (apêndice on-line).

Tabela 1 - Parâmetros florísticos e estruturais do cerradão e do cerrado típico com (CB) e sem (SB) agrupamentos de Actinocladum verticillatum no Parque Municipal do Bacaba, Nova Xavantina-MT. H' = Índice de diversidade de espécies de Shannon-Wiener. Valores entre parênteses são porcentagens.

\begin{tabular}{|c|c|c|c|c|c|c|}
\hline \multirow{2}{*}{ Parâmetro } & \multicolumn{3}{|c|}{ Cerradão } & \multicolumn{3}{|c|}{ cerrado típico } \\
\hline & SB & $\mathrm{CB}$ & Total & SB & $\mathrm{CB}$ & Total \\
\hline № de Indivíduos & 616 & 444 & 1060 & 970 & 268 & 1238 \\
\hline $\mathrm{N}^{\circ}$ de indivíduos mortos & $36(5,8)$ & $25(5,6)$ & - & $23(2,4)$ & $11(4,1)$ & - \\
\hline № registrado de espécies & 75 & 67 & 98 & 94 & 64 & 97 \\
\hline$N^{\circ}$ estimado de espécies ${ }^{1}$ & $103 \pm 4,57$ & $88 \pm 4,69$ & - & $105 \pm 3,67$ & $81 \pm 5,54$ & - \\
\hline № de Gêneros & 64 & 57 & 80 & 76 & 53 & 77 \\
\hline № de Famílias & 34 & 32 & 41 & 40 & 33 & 41 \\
\hline$N^{\circ}$ de espécies exclusivas ${ }^{2}$ & $31(41,3)$ & $23(34,3)$ & - & $28(29,8)$ & $3(4,5)$ & - \\
\hline $\mathrm{N}^{\circ}$ de espécies comuns ${ }^{2}$ & \multicolumn{2}{|c|}{44} & - & \multicolumn{2}{|c|}{60} & - \\
\hline Área basal (m²/0,15ha) & 3,528 & 3,012 & - & 3,285 & 2,115 & - \\
\hline H’ (nats/ind.) & 3,42 & 3,46 & - & 3,98 & 3,7 & - \\
\hline Índice de Pielou (J') & 0,79 & 0,82 & - & 0,88 & 0,88 & - \\
\hline Índice de Jaccard $\left(\mathrm{S}_{\mathrm{J}}\right)$ & \multicolumn{2}{|c|}{0,45} & - & \multicolumn{2}{|c|}{0,59} & - \\
\hline
\end{tabular}

Jackknife com intervalo de confiança de $95 \%$

2Comparação entre sítios (SB e CB) dentro da mesma fitofisionomia. 

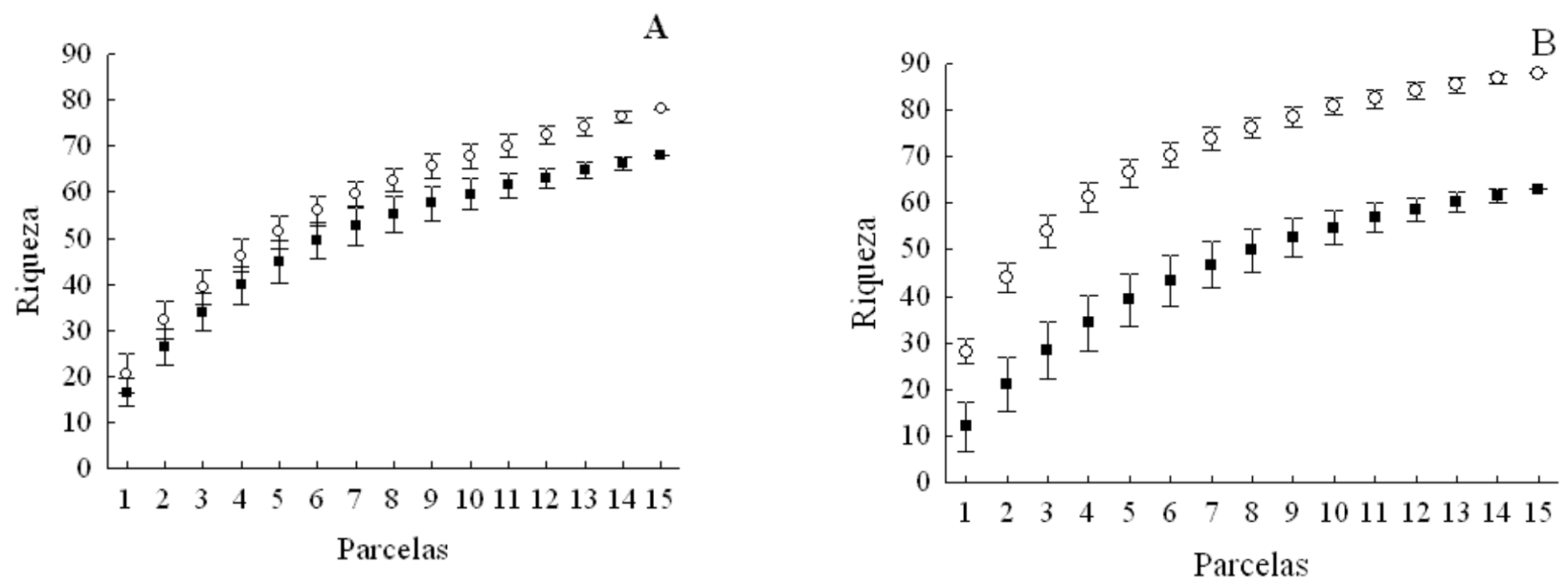

Figura 1 - Curva espécies-área baseada na aleatorização das amostras para o cerradão (A) e para o cerrado típico (B) em sítios com (๓) e sem (o) agrupamentos de Actinocladum verticillatum no Parque Municipal do Bacaba, Nova Xavantina-MT. Pontos centrais representam médias e barras verticais desvio padrão.

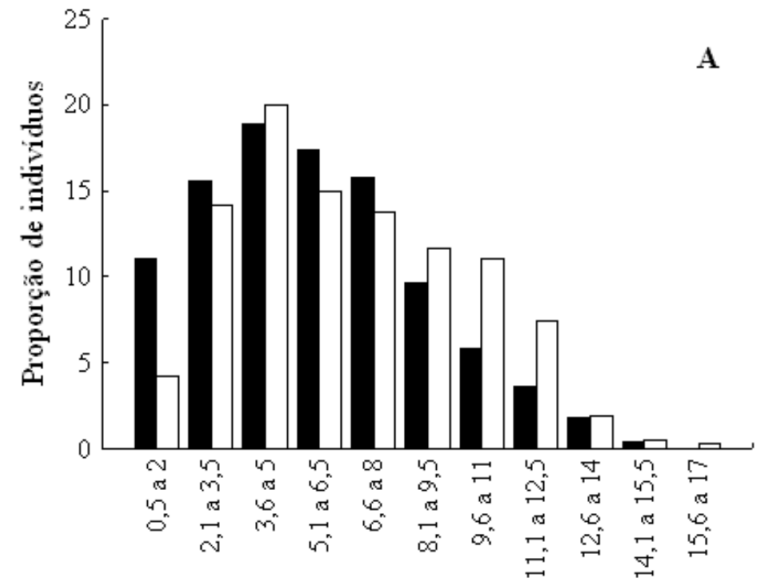

Classes de altura (m)

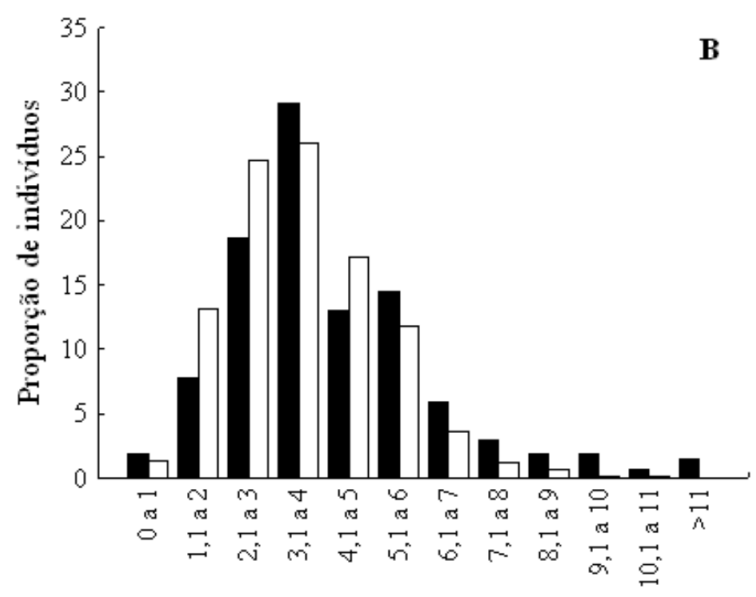

Classes de altura $(\mathrm{m})$

Figura 2 - Distribuição de alturas da vegetação lenhosa em sítios com (CB - barras negras) e sem agrupamentos (SB - barras brancas) de Actinocladum verticillatum no cerradão (A) e no cerrado típico (B) no Parque Municipal do Bacaba, Nova Xavantina-MT.

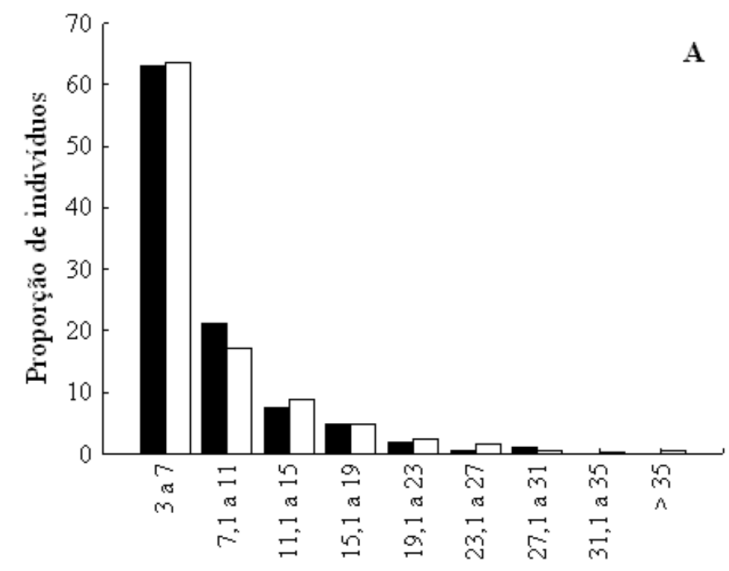

Classes de diâmetro $(\mathrm{cm})$

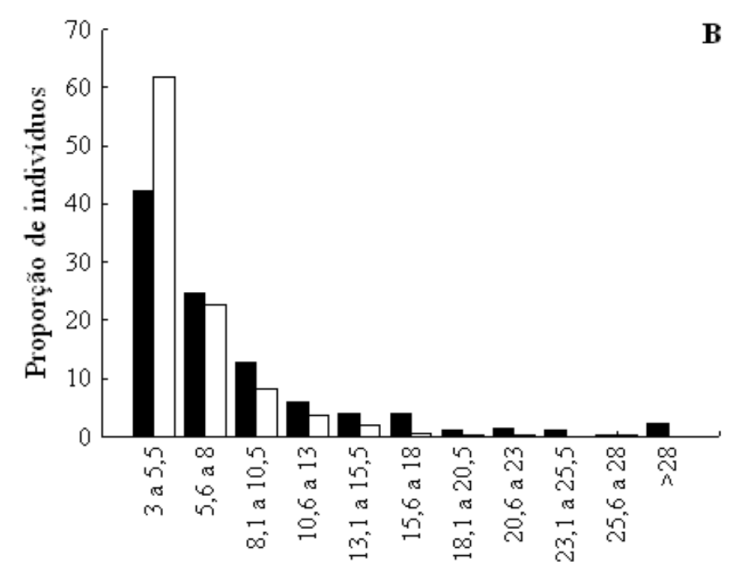

Classes de diâmetro $(\mathrm{cm})$

Figura 3 - Distribuição de diâmetros da vegetação lenhosa em sítios com (CB - barras negras) e sem agrupamentos (SB - barras brancas) de Actinocladum verticillatum no cerradão (A) e no cerrado típico (B) no Parque Municipal do Bacaba, Nova Xavantina-MT. 
No cerradão, apenas Sclerolobium paniculatum permaneceu entre as cinco mais importantes nos dois sítios, ocupando a primeira posição em ambos. No cerrado típico, não houve nenhuma coincidência entre as cinco espécies com maiores VI dos sítios $\mathrm{SB}$ e CB.

Quanto ao número de indivíduos, Myrcia sellowiana e Matayba guianensis estiveram sempre entre as cinco espécies mais abundantes nos sítios SB do cerradão e do cerrado típico; no entanto, foram representadas por um reduzido número de indivíduos nos sítios $\mathrm{CB}$ das duas fitofisionomias. Padrão semelhante foi observado para Heisteria ovata no cerradão (29 indivíduos no SB e ausente no $\mathrm{CB}$ ) e no cerrado típico (17 indivíduos no SB e 3 no $\mathrm{CB}$ ), bem como para Davilla elliptica (51 indivíduos no SB e um no CB) e Myrcia lanuginosa (30 indivíduos no $\mathrm{SB}$ e ausente no $\mathrm{CB}$ ) no cerrado típico (apêndice on-line).

Para as análises de classificação pelo método TWINSPAN foram excluídas 60 espécies com abundância inferior a cinco indivíduos, sem que nenhuma parcela fosse eliminada. A primeira divisão (autovalor $=0,334$ ) separou predominantemente as parcelas do sítio SB do cerradão dos três demais sítios, apontando Sclerolobium paniculatum e Hirtella glandulosa como espécies indicadoras e Xylopia aromatica, Myrcia sellowiana, Heisteria ovata e Tapirira guianensis como espécies preferenciais deste sítio. A segunda divisão (autovalor $=0,266)$ separou as parcelas do sítio SB do cerrado típico dos sítios CB do cerradão e CB do cerrado típico. Davilla elliptica foi apontada como espécie indicadora e Anacardium occidentale, Byrsonima pachyphylla, Mimosa laticifera, Myrcia lanuginosa e Rourea induta como preferenciais do sítio SB do cerrado típico. A terceira divisão (autovalor $=0,364$ ) separou os sítios CB do cerradão e CB do cerrado típico, apontando Roupala montana e Vatairea macrocarpa como espécies indicadoras do sítio CB do cerradão e Aspidosperma tomentosum e Lafoensia pacari como preferenciais do sítio CB do cerrado típico (Figura 4).

\section{DISCUSSÃO}

O fato da densidade e área basal nas duas fitofisionomias terem sido menores nos sítios $\mathrm{CB}$ pode estar relacionado à ocupação do espaço pelas touceiras de Actinocladum verticillatum, que dificulta a germinação e o estabelecimento das plantas lenhosas do Cerrado. Segundo Silveira (2001), o espaço é um recurso muito importante em florestas abertas da Amazônia, onde o bambu Guadua weberbaueri Pilger também apresenta intenso crescimento vegetativo, distribuição agrupada das touceiras e elevadas taxas de crescimento. Segundo este autor, a alta capacidade competitiva do bambu $G$. weberbaueri reduz a riqueza de espécies arbóreas e altera a estrutura e a dinâmica da vegetação. No Cerrado, a competição por luz (Nardoto et al. 1998; Braz et al. 2000; Franco 2002) e por água durante a estação seca, nas camadas mais superficiais do solo, com as raízes de gramíneas nativas (Hoffmann 1996; Franco 2002) podem ser fatores limitantes ao estabelecimento e à sobrevivência de plântulas de espécies lenhosas. Assim, tais fenômenos podem também estar ocorrendo nos sítios com Actinocladum verticillatum do presente estudo, nos quais as reduçóes na disponibilidade de água e luz, bem como no espaço disponível ao estabelecimento, diminuem as densidades e consequentemente a riqueza florística.

No cerrado típico do presente estudo os indivíduos de menor porte parecem realmente ser menos tolerantes à competição com Actinocladum verticillatum, uma vez que foi registrada maior frequência de indivíduos nas classes superiores de diâmetro e altura no sítio $\mathrm{CB}$ em relação ao sítio $\mathrm{SB}$. No entanto, os resultados para o cerradão não foram conclusivos em relação a este aspecto.

A menor diversidade de espécies observada no sítio CB do cerrado típico foi consequência da menor riqueza de espécies, uma vez que a equabilidade dos dois sítios foi igual. Segundo Magurran (1988) o índice de diversidade de Shannon-Wiener $\left(H^{\prime}\right)$ é sensível às variaçóes nas espécies raras. Dessa forma, a exclusão de espécies menos abundantes no sítio CB do cerrado

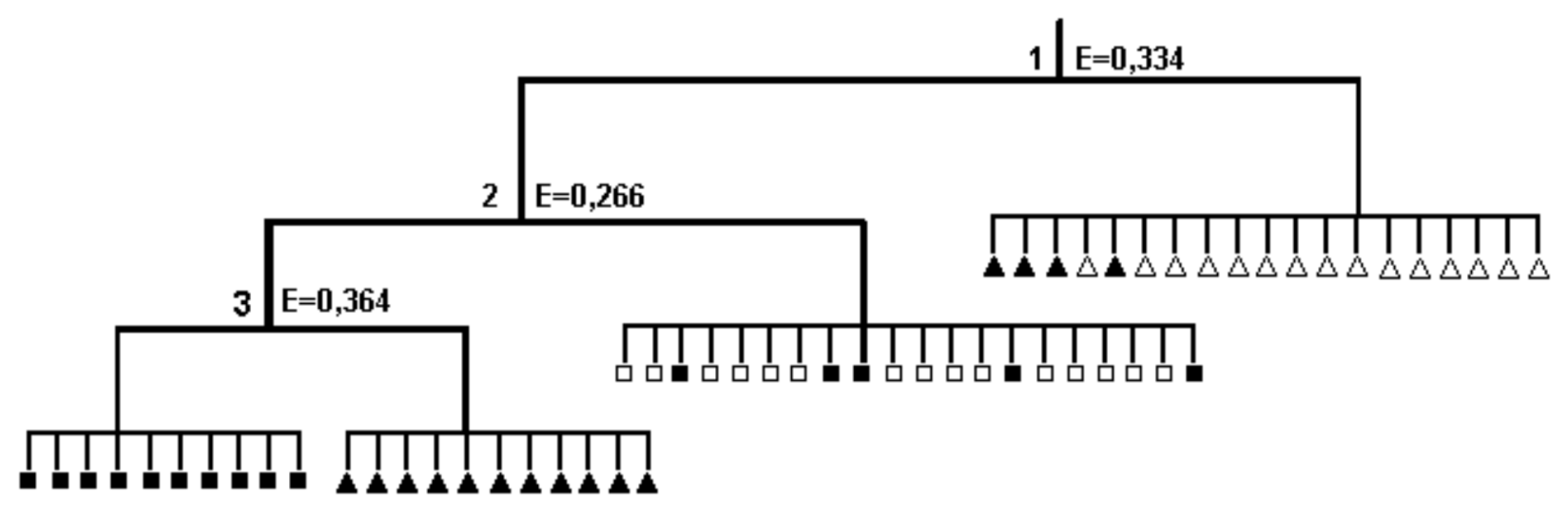

Figura 4 - Classificação das parcelas por meio da análise de TWINSPAN amostradas no cerradão com $(\boldsymbol{\Delta})$ e sem $(\Delta)$ agrupamentos de Actinocladum verticillatum e no cerrado com (๘) e sem (口) agrupamentos de A. verticillatum no Parque Municipal do Bacaba, Nova Xavantina-MT. 
típico também contribuiu para a baixa diversidade de espécies neste sítio. No cerradão, os valores de H' entre os dois sítios foram semelhantes, sugerindo um menor, ainda que evidente efeito do bambu sobre a riqueza e as abundâncias relativas das espécies. Estes resultados podem ser explicados pelo fato das plântulas de espécies lenhosas de formaçóes florestais do Cerrado apresentarem maior razáo de área foliar e investirem mais em crescimento do que as espécies de formaçóes savânicas (Hoffmann e Franco 2003), resultando numa maior facilidade para tolerar sombreamento.

Os sítios $\mathrm{SB}$ e $\mathrm{CB}$ dentro da mesma fitofisionomia apresentaram alta similaridade florística devido à proximidade entre eles ( $30 \mathrm{~m}$ no cerradáo e $20 \mathrm{~m}$ no cerrado típico) e as propriedades físico-químicas semelhantes do solo (Marimon Júnior e Haridasan 2005). Por outro lado, os sítios CB das duas fitofisionomias analisadas também apresentaram alta similaridade florística, sugerindo que a presença do bambu tem tornado áreas de cerradão e de cerrado semelhantes floristicamente.

Conforme verificado também por Marimon Júnior e Haridasan (2005), Sclerolobium paniculatum é uma espécie de grande importância no cerradáo. No presente trabalho, apenas esta espécie esteve entre as cinco espécies mais importantes em valor de importância (VI) nos dois sítios, deixando evidente que esta espécie é bastante resistente à competição, tanto com bambu quanto com as outras espécies arbóreas. Por outro lado, as espécies Myrcia sellowiana, Matayba guianensis e Heisteria ovata ocuparam posição de destaque em VI e apresentaram altas densidades locais no sítio SB, mas foram pouco representativas tanto em termos de VI quanto em número de indivíduos no sítio $\mathrm{CB}$, indicando que estas espécies são menos tolerantes a presença de bambu.

No cerrado típico estudado, a estrutura fitossociológica da comunidade foi também influenciada pelo agrupamento de bambu, pois quatro das cinco principais espécies em termos de VI e densidade do sítio SB, como Banisteriopsis pubipetala, Davilla elliptica, Myrcia sellowiana e Matayba guianensis, apresentaram baixa densidade e pequena representatividade em termos de VI no sítio CB. Desse modo, apesar da elevada similaridade florística entre os sítios SB e CB dentro da mesma fitofisionomia, alguns parâmetros estruturais da vegetação foram influenciados pela presença do bambu, devido à reduçấo na densidade de espécies pouco tolerantes à presença do bambu.

Outra evidência da distinçáo florística provocada pelo agrupamento do bambu foi dada pela classificação do TWINSPAN, na qual as duas primeiras divisóes separaram sítios SB dos sítios CB. Neste caso, a presença de Actinocladum verticillatum nas duas fitofisionomias pode estar atuando na seleção de espécies capazes de tolerar à forte pressão competitiva que o bambu exerce. A convergência florística entre os sítios $\mathrm{CB}$ de cerradáo e de cerrado típico, resultante da classificaçáo por TWINSPAN, pode estar relacionada à competição interespecífica das espécies lenhosas e do bambu. Segundo Townsend et al. (2006), a competição por recursos pode excluir uma ou mais espécies de sítios onde estas poderiam se estabelecer na ausência de competição. Assim sendo, a exclusão ou redução na densidade de espécies sensíveis à presença do bambu no cerrado típico e no cerradáo pode ter aumentado a similaridade florística entre as duas fitofisionomias.

Segundo Soderstrom (1981), Actinocladum verticillatum está adaptada a regenerar-se com rapidez após o fogo, multiplicando-se vegetativamente e resultando em uma maior densidade de ramos e de folhagem. Dessa forma, as queimadas no cerrado podem se intensificar com o aumento de material combustível produzido por bambus (Silveira 2001), potencializando os efeitos do fogo sobre a composiçấo e riqueza florística e na estrutura e dinâmica da vegetação lenhosa. Dois meses após a realização do presente estudo ocorreu uma intensa queimada nos dois sítios ( $\mathrm{SB}$ e $\mathrm{CB}$ ) de cerrado típico estudados, abrindo a oportunidade para a condução de novos estudos testando o efeito integrado da presença de $A$. verticillatum e do fogo nesta fitofisionomia.

A região de transição Cerrado-Floresta Amazônica vem sofrendo um aumento na freqüência de incêndios (Nepstad $e t$ al. 1999; Fearnside 2005), fato que, em longo prazo, poderá ocasionar um incremento das densidades das populaçóes de A. verticillatum na regiāo (Soderstrom 1981), podendo afetar direta e indiretamente as estrutura das comunidades de plantas lenhosas. Nesse sentido, é de suma importância que estudos de monitoramento dos agrupamentos do bambu $A$. verticillatum sejam realizados com a finalidade de compreender e manejar sua distribuição na regiấo.

Quando considerados em conjunto, os resultados do presente estudo sugerem que os agrupamentos de Actinocladum verticillatum reduzem a densidade e a área basal e alteram a composição florística e a estrutura vertical e horizontal do componente lenhoso das duas fitofisionomias estudadas. Conforme esperado, os efeitos da presença do bambu foram mais evidentes no cerrado típico, um ambiente savânico e heliófilo, uma vez que, nessa fitofisionomia, houve ainda redução na riqueza e diversidade de espécies e forte alteração na estrutura diamétrica e de alturas da comunidade no sítio CB. Assim, a comunidade lenhosa do cerrado típico é menos tolerante do que a do cerradão em relação aos efeitos provocados por aumentos nas densidades de $A$. verticillatum. No entanto, os mecanismos responsáveis por estes processos (alelopatia, competição por espaço, luz, água e nutrientes) nas duas fitofisionomias são ainda desconhecidos e aguardam estudos mais específicos para sua confirmação. 


\section{AGRADECIMENTOS}

A CAPES pela concessão de bolsa de Pós-Graduação aos dois primeiros autores, aos Biólogos Edmar A. Oliveira, Leandro S. Silva e Claudinei O. Santos pela valiosa ajuda durante as coletas de campo.

\section{BIBLIOGRAFIA CITADA}

Abad, J.C.S.; Marimon, B.S. 2008. Characterization and environmental diagnosis of the Municipal Bacaba Park (Nova Xavantina, MT). In: Santos, J.E.; Galbiati, C. (Orgs.). Management and environmental education: water, biodiversity and culture. Rima, São Carlos. p. 2356 (in Portuguese, with abstract in English).

APG II - Angiosperm Phylogeny Group. 2003. An update of the Angiosperm Phylogeny Group Classification for the orders and families of flowering plants: APG II. Botanical Journal of the Linnean Society, 141(4): 399-436.

Braz, V.S.; Kanegae, M.F.; Franco, A.C. 2000. Establishment and development of Dalbergia miscolobium Benth. in two vegetation types typical of the savannas of central Brazil. Acta Botanica Brasilica, 14(1): 27-35 (in Portuguese, with abstract in English).

Brower, J.E.; Zar, J.H. 1977. Field and laboratory methods for general ecology. W.C. Brown Co. Pub., Iowa.

Camargo, A.P. 1963. Cerrado Climate. In: Ferri, M.G. (coord.). Sympósio do Cerrado. EDUSP, São Paulo. p. 75-59 (in Portuguese).

Cientec. 2002. Mata Nativa 2: versão 2.0. Cientec, Viçosa (in Portuguese).

Colwell, R.K. 2008. EstimateS: Statistical estimation of species richness and shared species from samples. Version 8.0. (http://www.purl. oclc.org/estimates). Acessed: 15/10/08.

Curtis, J.T.; McIntosh, R.P. 1950. The interrelations of certain analytic and synthetic phytosociological characters. Ecology, 31(3): 434-455.

Curtis, J.T.; McIntosh, R.P. 1951. An upland forest continuum in the prairie-forest border region of Wisconsin. Ecology, 32(3): 476-946.

Fearnside, P.M. 2005. Deforestation in the Brazilian Amazon: history, rates and consequences. Megadiversidade, 1(1): 113-123 (in Portuguese, with abstract in English).

Felfili, J.M.; Carvalho, F.A.; Libano, A.M.; Venturoli, F.; Pereira, B.A.S. 2007. Multivariate analysis in studies of vegetation. Comunicaçôes Técnicas Florestais da UnB, 9: 1-60 (in Portuguese).

Filgueiras, T.S. 1988. Native forage Grass species from the Federal District, Brazil (Gramineae: Bambusoideae). Revista Brasileira de Botânica, 11: 47-66 (in Portuguese, with abstract in English).

Franco, A.C. 2002. Ecophysiology of woody plants. In: Oliveira, P.S.; Marquis, R.J. (Eds.). The Cerrados of Brazil. Columbia University Press, New York. p. 178-197.

Gotelli, N.J.; Entsminger, G.L. 2001. EcoSim: Null models software for ecology. Version 7.0. Acquired Intelligence Inc. \& Kesey-Bear.

Hill, M.O. 1979. TWINSPAN - A fortran program for arranging multivariate data in an ordered two-way table by classification of individuals and attributes. Cornell University, New York.
Hoffmann, W.A.; Franco, A.C. 2003. Comparative growth analysis of tropical forest and savanna woody plants using phylogenetically independent contrasts. Journal of Ecology, 91(3): 475-484.

Hoffmann, W.A. 1996. The effects of fire and cover on seedlings establishment in a neotropical savanna. Journal of Ecology, 84(3): 383-393.

Ivanauskas, N.M.; Monteiro, R.; Rodrigues, R.R. 2004. Floristic composition of forest patches in southern Amazonia. Acta Amazonica, 34(2): 281-305 (in Portuguese, with abstract in English).

Janzen, D.H. 1976. Why bamboos wait so long to flower. Annual Review of Ecology, Evolution and Systematics, 7: 347-391.

Kent, M.; Coker, P. 1992. Vegetation description and analysis; a practical approach. Bealhaven Press, London.

Krebs, C.J. 2000. Ecological Methodology. 2 ed. Harper and Row Publishers, New York.

Ludwing, J.A.; Reynolds, J.E. 1988. Statistical ecology: a primer on methods and computing. J. Wiley e Sons, New York.

Magurran, A.E. 1988. Ecological diversity and its measurement. Croom Helm, London.

Marimon Júnior, B.H.; Haridasan, M. 2005. A comparison of the woody vegetation and soil characteristics of a cerradão and a Cerrado sensu stricto in adjacent areas on dystrophic soils in eastern Mato Grosso State, Brazil. Acta Botanica Brasilica, 19(4): 913-926 (in Portuguese, with abstract in English).

Marimon, B.S.; Felfili, J.M. 2006. Seed rain in a monodominant Brosimum rubescens Taub. forest and an adjacent mixed forest in the Araguaia River Valley, Mato Grosso State, Brazil . Acta Botanica Brasilica, 20(2): 423-432 (in Portuguese, with abstract in English).

Marimon, B.S.; Lima, E.S.; Duarte, T. G.; Chieregatto, L. C.; Ratter, J. A. 2006. Observations on the vegetation of northeastern Mato Grosso, Brazil. IV. An analysis of the Cerrado-Amazonian Forest ecotone. Edinburgh Journal of Botany, 63(2,3): 323-341.

Mendonça, R.C; Felfili, J.M.; Walter, B.M.T.; Silva Júnior, M.C.; Rezende, A.V.; Filgueiras, T.S.; Nogueira, P.E.; Fagg, C.W. 2008. Vascular Flora of the Cerrado biome. In: Sano, S.M.; Almeida, S.P.; Ribeiro, J.F. (Eds.). Cerrado: ecology and flora. Embrapa Informação Tecnológica, Brasília. p.421-1.279. (in Portuguese).

Mobot - Missouri Botanical Garden. 2009. W3 Trópicos. (http:// www.mobot.org/w3t/search/vast.htm). Acessed: 20/03/09.

Müller-Dombois, D.; Ellemberg, H. 1974. Aims and methods of vegetation ecology. J. Wiley \& Sons, New York.

Nardoto, G.B.; Souza, M.P.; Franco, A.C. 1998. Establishment and seasonal patterns of growth in Kielmeyera coriacea (Spr) Mart. in the cerrados of Central Brazil: effects of water stress and shading. Revista Brasileira de Botânica, 21(3): 313-319 (in Portuguese, with abstract in English).

Nepstad, D. C.; Moreira, A.; Alencar, A.A. 1999. Forest in flame: Origins, Impact and Prevention of Fire in the Amazon. Pilot Program to Conservation of the Rain Forest of Brazil, Brasilia, Brazil (in Portuguese). 
Nogueira, E.M.; Nelson, B.W.; Fearnside, P.M.; França, M.B.; Oliveira, A.C.A. 2008. Tree height in Brazil's "arc of deforestation": shorter trees in south and southwest Amazonia imply lower biomass. Forest Ecology and Management, 255: 2.963-2.972.

Oliveira-Filho, A.T.; Vilela, E.A.; Gavilanes, M.L.; Carvalho, D.A. 2004. Effect of flooding regime and understorey bamboos on the physiognomy e tree species composition of a tropical semideciduos forest in Southeastern Brasil. Vegetatio, 113(2): 99-124.

Ratter, J.A.; Richards, P.W.; Argent, G.; Gifford, D.R. 1973. Observations on the vegetation of the northeastern Mato Grosso. I. The Woody vegetation types of the Xavantina-Cachimbo Expedition area. Philosophical Transactions of the Royal Society of London, 266: 449-492.

Ribeiro, J.F.; Walter, B.M.T. 2008. The main vegetation physiognomies of the Cerrado. In: Sano, S.M.; Almeida, S.P.; Ribeiro, J.F. (Eds.). Cerrado: ecology and flora. Embrapa Informação Tecnológica, Brasília. p. 151-212 (in Portuguese).

Sanquetta, C.R.; Dalla Corte, A.P.; Vulcanis, L., Berni, D.M.; Biscaia, A.G. 2006. Establishment of tree seedlings species in an experiment of bamboos (Bambusoideae) control in southern Paraná, Brazil. Revista Ciências Exatas e Naturais, 8(2): 163-177 (in Portuguese, with abstract in English).
Scolforo, J.R.S. 1993. Measurement Forestry 5: Forest growth 1. ESAL/ FAEPE, Lavras (in Portuguese).

Siegel, S.; Castellan-Júnior, N.T. 1988. Nonparametric statistics for the behavioral sciences. McGraw-Hill, New York.

Silveira, M. 2001. Open forests with bamboos of the southwestern Amazonia: patterns and processes at multiple scales. Tese de doutorado, Universidade de Brasília, Brasília. 121 pp (in Portuguese).

Soderstrom, T.R. 1981. Observations on fire-adapted bamboo of the Brazilian Cerrado, Actinocladum verticillatum (Poaceae: Bambusoidae). American Journal of Botany, 68(9): 1200-1211.

Spiegel, M.P. 1976. Statistics. McGraw-Hill, São Paulo. (in Portuguese).

Townsend, C.R.; Begon, M.; Harper, J.L. 2006. Fundamental ecology. Artmed, Porto Alegre (in Portuguese).

Zar, J.H. 1999. Biostatistical analysis. Prentice Hall, New Jersey.

Recebido em 07/10/2009

Aceito em 09/12/2009 
